\title{
Le leadership de l'Église en Acadie (1864-1942)
}

\section{Fernand Arsenault}

Volume 50, numéro 2, 1983

Bilan de l'histoire religieuse au Canada

Canadian Catholic History: A survey

URI : https://id.erudit.org/iderudit/1007216ar

DOI : https://doi.org/10.7202/1007216ar

Aller au sommaire du numéro

Éditeur(s)

Les Éditions Historia Ecclesiæ Catholicæ Canadensis Inc.

ISSN

0318-6172 (imprimé)

1927-7067 (numérique)

Découvrir la revue

Citer cet article

Arsenault, F. (1983). Le leadership de l'Église en Acadie (1864-1942). Sessions d'étude - Société canadienne d'histoire de l'Église catholique, 50(2), 453-470.

https://doi.org/10.7202/1007216ar

Tous droits réservés @ Les Éditions Historia Ecclesiæ Catholicæ Canadensis Inc., 1983
Ce document est protégé par la loi sur le droit d'auteur. L'utilisation des services d'Érudit (y compris la reproduction) est assujettie à sa politique d'utilisation que vous pouvez consulter en ligne.

https://apropos.erudit.org/fr/usagers/politique-dutilisation/ 


\section{Le leadership de l’Église en Acadie (1864-1942)}

$\mathrm{Au}$ moment de l'ouverture du Collège St-Joseph, en 1864, la population acadienne se trouvait dans un état lamentable sur tous les plans. La pauvreté était profonde. L'agriculture, la pêche et la forêt permettaient tout simplement de subsister. L'éducation primaire et secondaire se voyait dévalorisée et peu accessible à la grande majorité. Jusque vers 1912, les Acadiens ne jouissaient que de peu de pouvoir dans la société civile comme dans l'Église, malgré qu'ils formaient plus de la moitié de la population catholique dans trois diocèses des provinces Maritimes. Tout était à faire ${ }^{1}$. Et les obstacles semblaient presque insurmontables: opposition farouche de la part des autorités religieuses et politiques; manque de confiance, beaucoup de peur et de timidité chez une population démunie et écrasée depuis plus d'un siècle.

Faut-il se surprendre que le leadership acadien ne foisonnait pas à l'époque. On connaissait le travail des abbés Hubert Girroir dans la région d'Arichat - Cheticamp, Georges-Antoine Belcourt sur l'Île du Prince-Édouard, Antoine Gagnon dans la région de Shédiac, FrançoisXavier Lafrance dans la paroisse de Tracadie et de Memramcook, Jean Mandé Sigogne dans la Baie Ste-Marie et Antoine Langevin à StBasile et au Madawaska. Mais dans le coeur de plusieurs jeunes comme Marcel-François Richard, Stanislas Doucet, Philippe Belliveau, PierreAmand Landry, Pascal Poirier, de plus en plus éveillés par leur milieu familial aux injustices que subissaient leurs concitoyens, montait un grand rêve de libération nationale ${ }^{2}$.

N.B.- Je tiens à remercier Madame Phyllis Roy, secrétaire, pour son indispensable contribution. Également mes collègues Léon Thériault et Edmour Babineau qui ont bien voulu lire cette étude et me faire part de leurs précieux commentaires.

'Léon Thériault, «L'acadianisation de l'Église en Acadie, 1763-1953", in Les Acadiens des Maritimes, Moncton, Centre d'études acadiennes, 1980, p. 340.

Calixte Savoie, Mémoire d'un nationaliste acadien, Moncton, Ed. d'Acadie, 1979, p. 38-9.

${ }^{2}$ Camille-Antonio Doucet, Une étoile s'est levée en Acadie - Marcel-François Richard, Charlesbourg-Est, Éd. du Renouveau, 1973, p. 18. 
Mais quel a été le véritable impact de l'Église catholique dans ces années de luttes et de reconstruction de la nation acadienne? Selon certains, l'Église catholique a exercé dans cette période un leadership négatif qui a contribué largement à la dépersonnalisation des Acadiens, à leur enracinement dans la soumission et la peur, les empêchant ainsi de se réaliser comme peuple. "Cette élite apathique et rétrograde (clergé et petite élite laïque) prêchera le conservatisme à outrance alors qu'on est en pleine période d'industrialisation" ${ }^{3}$. Cette thèse m'apparaît peu respectueuse de la réalité historique dans son ensemble.

Il faut admettre qu'à la fin du XIX ${ }^{\mathrm{e}}$ siècle l'Église catholique en Acadie, tout comme au Québec et ailleurs dans le monde, a connu de longues années de repliement sur elle-même. Afin de préserver les Acadiens des dangers du protestantisme, du modernisme et de la société urbaine et industrielle, l'Église catholique est devenue très cléricale, triomphaliste et autoritaire. Sa prédication est livresque et son langage peu adapté aux préoccupations d'une population peu scolarisée. Le mariage, l'amour humain et la vie dans le monde sont dévalorisés au bénéfice de la vie religieuse et sacerdotale. Trop souvent la peur de l'enfer remplace l'amour de Dieu comme motivation à l'observance des commandements. De nombreux textes de prédicateurs et plusieurs témoignages de chrétiens viennent confirmer ces faits. Par contre, si nous analysons l'enseignement que nous ont laissé les principaux porteparole acadiens de 1864 à $1942^{4}$, et l'ensemble de leurs réalisations, force nous est de constater le caractère hautement bénéfique de l'oeuvre du clergé, de l'épiscopat et du laïcat chrétien dans la renaissance acadienne.

\footnotetext{
${ }^{3}$ Régis Brun, De Grand-Pré à Douchibouguac - L'histoire d'un peuple exploité, Moncton, Ed. d'Acadie, 1982, p. 147.

Michel Roy, L'Acadie perdue, Montréal, Éd. Québec/Amérique, 1978.

${ }^{4} \mathrm{~J}$ 'ai choisi de limiter cette étude à la période allant de 1864 à 1942 , pour répondre autant que possible à l'invitation de la Société canadienne d'histoire de l'Église catholique. De plus, pour les Acadiens, 1864 rappelle l'ouverture du Collège St-Joseph et le début de ce que l'on a appelé la Renaissance acadienne. Je termine avec 1942, l'année du décès de $\mathrm{M}^{\mathrm{gr}}$ Patrice-Alexandre Chiasson, évêque de Bathurst. Cette date représente la fin de la première génération d'évêques acadiens. $\mathrm{M}^{\mathrm{gr}}$ Edouard LeBlanc, premier évêque acadien, était décédé le 18 février 1935 et $\mathrm{M}^{\mathrm{gr}}$ Arthur Melanson, premier archevêque de Moncton, le 30 octobre 1941.
} 


\section{COMMENT L'ÉGLISE PERCEVAIT-ELLE SA MISSION EN ACADIE?}

L'Église catholique a été très longtemps identifiée à la cause du peuple acadien. Pour le clergé comme pour le laïcat, sa mission spirituelle se coulait naturellement dans le projet global de ce petit peuple qui avait juré de ne pas mourir et de prendre toute sa place au soleil. Pour $\mathrm{M}^{\mathrm{gr}}$ Stanislas Doucet, curé à Pokemouche, Shippagan et GrandAnse dans le nord-est du Nouveau-Brunswick, entre 1870 et 1925, égalité, liberté et fraternité pour tous est la devise de l'Église en Acadie comme ailleurs dans le monde, et les membres du clergé acadien entendaient bien mettre tout en oeuvre pour réaliser chez leurs compatriotes des Provinces Maritimes ce défi révolutionnaire.

C'est le bien, c'est l'avancement de la grande famille humaine sous tous les rapports qu'ils |les membres du clergé $\mid$ désirent, qu'ils ambitionnent, et ils emploient tous les moyens propres à les aider dans leur travail de dévouement et de sacrifice... La vigne du Seigneur est vaste, et la moisson abondante. De tous côtés il y a du bien à faire, et tout peut coopérer au bien... En avant! et les ministres de la vraie religion s'élancent dans toutes les voies qui mènent au véritable progrès... En avant! et ils explorent le vaste champ des connaissances humaines. Les sciences, les lettres et les arts trouvent en eux leurs plus zélés promoteurs; l'éducation de la jeunesse, son plus ferme appui. Rien de tout ce qui peut contribuer en aucune manière au bien spirituel et temporel de l'humanité n'est étranger aux ouvriers évangéliques. Égalité, liberté, fraternité, - dans le vrai sens, le sens évangélique des termes, - voilà leur devise. $^{5}$

Selon $\mathrm{M}^{\mathrm{gr}}$ Doucet, Marcel-François Richard, le curé de Rogersville, était un témoin de cette race d'hommes qui ont voué leur vie au mieuxêtre des Acadiens. Et ce mieux-être, c'est l'objectif que semble bien s'être donné tous les leaders de l'époque. Nous retrouvons le même discours chez le Père François Bourgeois, né à Cocagne au NouveauBrunswick en 1886, pour qui le prêtre apporte à la société acadienne de nombreux et riches bienfaits temporels et une contribution indispensable à son épanouissement ${ }^{6}$. Et les trois premiers évêques acadiens, inspirés par l'enseignement social de Léon XIII, et le Pie XI, n'ont certai-

\footnotetext{
${ }^{5} \mathrm{M}^{\mathrm{gr}}$ Stanislas-J. Doucet, «Ce que l'Église catholique a fait pour le bien général de la société", sermon donné à Rogersville, le 15 août 1895, à l'occasion des noces d'argent de M. l'abbé Marcel-François Richard, Le Moniteur Acadien (MA), 27 août 1895, p. 1.

${ }^{6}$ L'Évangéline (EV.), 30 octobre 1930.
} 
nement pas contredit l'oeuvre amorcée par les pionniers du $\mathrm{XIX}^{\mathrm{e}}$ siècle. Tout en se faisant foncièrement pasteurs d'âmes, ils ont recommandé à plusieurs reprises à leurs prêtres et collaborateurs laïques, de voir comme partie de leur apostolat tout ce qu'ils feraient pour l'amélioration de la vie sociale et économique de ceux qui leur étaient confiés ${ }^{7}$.

En lisant les discours et les écrits de ces bâtisseurs acadiens, on sent qu'il y a beaucoup à faire et que l'Acadie est en train de devenir un grand chantier. En l'absence d'un pouvoir laïque acadien bien défini et dans le contexte d'une église autoritaire, on ne s'étonnera pas de ce que le leadership du clergé ait été partout présent et qu'il se soit souvent exercé de façon quelque peu militaire. Les évêques comptent sur la prompte et généreuse obéissance de tous les fidèles... «laquelle devra se prouver dans des actes et une coopération entière et constante que nous sommes en droit d'attendre de chacun et de tous... Sacrifice peutêtre de vos idées personnelles; sacrifice sûrement de vos personnes... sacrifice encore non moins impérieux et nécessaire de vos aumônes". Pour sortir du marasme dans lequel l'Acadie s'enlisait depuis plus d'un siècle se met sur pieds un grand mouvement d'actions souvent centralisé et coordonné à partir du "trône" de l'évêque ${ }^{8}$.

\section{QUATRE MOTS D'ORDRE NATIONAUX}

Nous ne reprendrons pas dans le détail la longue liste des réalisations du clergé et des communautés religieuses en Acadie. Ce travail a déjà été fait, du moins en partie. Dans les pages qui vont suivre nous ferons ressortir les grands axes de la contribution de l'Église en Acadie en nous basant sur les interventions écrites des principaux leaders. En parcourant ces textes, nous avons relevé quatre grands appels au peuple acadien. Ces mots d'ordre ou défis ont sans aucun doute inspiré profondément les réalisations des Acadiens depuis plus d'un siècle. De 1864 à 1942, des membres influents du clergé et du laïcat ont cherché sans relâche à valoriser l'éducation et à réveiller la fierté nationale chez les Acadiens; ils les ont poussés à devenir autonomes et maîtres chezeux, à sortir des vieux sentiers et à moderniser leur économie. Voyons maintenant chacun de ces mots d'ordre afin d'en évaluer la force et l'impact.

\footnotetext{
${ }^{7} \mathrm{M}^{\mathrm{gr}}$ Édouard Leblanc, Lettre circulaire (L.C. ) 28 janvier 1913;

$\mathrm{M}^{\mathrm{gr}}$ Patrice-Alexandre Chiasson, Lettre circulaire (L.C. ) no 13, 1935;

$\mathrm{M}^{\mathrm{gr}}$ Arthur Melanson, Lettre pastorale (L.P.), 22 février 1937.

${ }^{8} \mathrm{M}^{\mathrm{gr}}$ Arthur Melanson, L.P., 22 février 1937.
} 


\section{L'éducation: notre salut}

Les premiers gestes de relèvement national en Acadie ont porté sur l'éducation. Prêtres et laïques ont vite réalisé que leur salut dépendrait de la rapidité avec laquelle ils mettraient l'éducation au service de tous les Acadiens. Dès 1840, l'abbé Antoine Gagnon travaillait à l'établissement d'un collège dans la région de Shédiac et l'abbé François-Xavier Lafrance ouvrit une première maison d'enseignement supérieur à Memramcook en $1854^{9}$. Mais pour un peuple qui vivait dans une extrême pauvreté depuis plus d'un siècle, les obstacles étaient nombreux: l'instruction au primaire avait été nécessairement négligée et cheż de larges couches de la population "des préjugés invétérés... font croire qu'il est inutile de s'occuper de la culture intellectuelle" ${ }^{10}$. Il faut donc tout mettre en oeuvre pour combattre les préjugés des Acadiens face à l'école et chercher à valoriser l'éducation et la profession d'enseignant. À la première Convention Nationale, les Acadiens se proposent donc de mieux payer leurs instituteurs, d'embellir leurs écoles et de veiller à ce que la pédagogie respecte davantage la personnalité de l'enfant. Le Père Philias Bourgeois, c.s.c., invite alors les Acadiens à se doter d'une école où l'enseignement sera simple, utilitaire et intuitif.

C'est ce mode d'enseignement qui donne à l'école moderne ses deux caractères distinctifs; d'une part un certain aspect aimable et presque gai, des études qui se font presque en jouant, une école où l'enfant se plaît, une éducation d'où l'effort et la contrainte sont bannis; d'une autre part, ce second caractère, non moins frappant, c'est que tout l'enseignement est pratique, usuel: on n'apprend aux enfants que ce dont ils auront à se servir. ${ }^{11}$

Et c'est encore à l'occasion de cette première Convention que le Père Philias Bourgeois et les membres de là Commission de l'éducation (dans laquelle figuraient le Père Lefebvre, l'abbé Biron du Collège St-

\footnotetext{
${ }^{9}$ Le collège de l'abbé Antoine Gagnon ne vit jamais le jour faute de ressources financières et de collaboration de la part de son évêque, $\mathrm{M}^{\mathrm{gr}}$ Donald Macdonald, de Charlottetown. Cf. Léon Thériault, «L'acadianisation de l'Église catholique en Acadie, 1763-1953», in Les Acadiens des Maritimes, Centre d'Études Acadiennes, Moncton, 1980, p. 313. Quant au collège de l'abbé F.-X. Lafrance, il fut repris en 1864 par la Congrégation de Ste-Croix et devint le Collège puis I'Université Saint-Joseph qui, en 1963, se fusionna avec d'autres institutions pour donner naissance à l'Université de Moncton.

${ }^{10}$ Philias F. Bourgeois, c.s.c., Rapport sur l'éducation, in Conventions nationales des Acadiens, vol. 1, Shediac, N.-B., 1907, p. 116.

${ }^{11}$ Philias F. Bourgeois, c.s.c., Ibid., p. 119;

$\mathrm{M}^{\mathrm{gr}}$ Patrice-Alexandre Chiasson, Sermon prononcé à la messe du $2^{e}$ Congrès de l'Association Acadienne d'Éducation (A.A.E.) à Memramcook, le 5 août 1940.
} 
Louis, Le Père Stanislas Doucet, le journaliste Valentin A. Landry, et le Père $\mathrm{H}$. Girroir) proposèrent aux Acadiens de viser à préparer la jeunesse à toutes les positions commerciales et industrielles du jour tout en respectant les aptitudes de chacun.

Il faut... les | les jeunes Acadiens | initier de bonne heure à l'étude des lois et des phénomènes qui ont amené les progrès gigantesques du monde industriel, et jeté tant de lumière au fond de toutes les questions de l'économie humaine.

Et ce travail, où se fera-t-il? à l'école. Le dessin, l'architecture, le génie civil, tout ce qui se rattache au commerce, aux finances, aux exploitations manufacturières, aux détails intimes de l'économie domestique. En joignant ainsi, dans l'enseignement, l'étude des principes avec la pratique ou avec la science appliquée, l'élève saura quelle direction imprimer à ses travaux, l'école deviendra alors un atelier, le maitre apprendra à son élève à manier les instruments de son travail et il se fera artisan, c'est-à-dire artisan de son avenir, de sa destinée. ${ }^{12}$

Cet audacieux projet de 1881 fera très lentement son chemin dans les institutions acadiennes d'éducation. La pauvreté des ressources financières et le manque criant de personnel ont sans doute empêché ces éducateurs réalistes d'atteindre plus rapidement leur grand objectif. Mais les leaders acadiens ne manquèrent jamais une occasion de rappeler à la population que leur avenir dépendait de leurs efforts à promouvoir l'éducation.

Instruisons-nous, Mes Frères. Notre influence dans ce pays sera proportionnée à notre progrès dans l'éducation. Les autres nationalités ont pris les devants; c'est qu'elles sont plus instruites. Ce qu'elles ont fait nous pouvons le faire aussi. Nous sommes aussi intelligents qu'eux. Ne craignons donc pas de dépenser quelques centaines de piastres pour l'éducation de nos enfants. Remplissons nos collèges d'élèves intelligents et avides de science et l'avenir de la race acadienne est assuré. ${ }^{13}$

Pour $\mathbf{M}^{\mathrm{gr}}$ Philippe Belliveau, comme pour $\mathrm{M}^{\mathrm{gr}}$ Marcel-François Richard, c'est l'éducation et l'éducation seule qui nous donnera les citoyens capables d'administrer nos affaires et de défendre nos droits ${ }^{14}$.

\footnotetext{
${ }^{12}$ Philias F. Bourgeois, c.s.c., Ibid., p. 120.

${ }^{13}$ A.E. Monbourquette, curé de Magarie, Nouvelle-Écosse, Sermon prononcé le 15 août 1902, MA, 11 septembre 1902, p. 1.

${ }^{14} \mathrm{M}^{\mathrm{gr}}$ Philippe Belliveau, Centre d'Études Acadiennes (CEA), Fonds, 24.3-2, p. 13;

$\mathrm{M}^{\mathrm{gr}}$ Marcel-François Richard, 15 août 1890, in Conventions Nationales des Acadiens, T. I, p. 240-57.
} 
Et cette éducation elle devra tendre au développement intégral de toutes les facultés des étudiants - «les facultés physiques par un exercice bien ordonné, les facultés intellectuelles par une solide instruction, où la science et la religion s'harmonisent, et où les facultés morales reçoivent une attention toute particulière par la formation du caractère et de la volonté...» '5

Pour leur part, $\mathbf{M}^{\mathrm{gr}}$ Patrice-Alexandre Chiasson, évêque de Chatham au Nouveau-Brunswick de 1920 à 1942 et $\mathbf{M}^{\text {gr }}$ Arthur Melanson, nommé premier archevêque de Moncton en 1936, dès leur arrivée en Acadie, ont mis tout le poids de leur autorité épiscopale pour seconder la cause de l'éducation. $\mathbf{M}^{\mathrm{gr}}$ Chiasson a fortement invité les parents à valoriser l'éducation dans leur milieu, en mettant tout en oeuvre pour doter l'école d'instituteurs et d'institutrices bien rémunérés et compétents à tous points de vue, et en faisant de l'école «la plus belle maison du village. Elle sera propre, bien tenue et attrayante" ${ }^{16}$. Il les engage également à considérer comme un devoir sacré de payer leurs taxes scolaires et de veiller à ce que leurs enfants reçoivent l'enseignement dans la langue maternelle. Selon $\mathrm{M}^{\mathrm{gr}}$ Chiasson, l'école se doit de respecter les moeurs et le génie propre à chacun. "Vos enfants, dit-il, sont des Acadiens-français et à ce titre on doit leur enseigner leur langue... On ne dispense pas la vérité et la science de la même manière aux Français et aux Anglais» ${ }^{17}$. Et pour que les élèves puissent bénéficier au maximum de l'école, les parents devront demeurer responsables de l'éducation de leurs enfants, en collaboration avec les enseignants qui ne sont que leurs représentants.

$\mathbf{M}^{\mathrm{gr}}$ Arthur Melanson a fait siennes les directives de $\mathbf{M}^{\mathrm{gr}}$ Chiasson en matière d'éducation; il a invité tous ses fidèles à se regrouper en cercles d'études ou en famille pour les lire et les approfondir. "Tout le monde déplore l'apathie, l'indifférence, voir même la négligence d'un trop grand nombre de parents vis-à-vis de la petite école. Il faut à tout prix les éclairer et leur donner une direction ferme. Voilà la grande mission qui nous est échue» ${ }^{18}$.

${ }^{15} \mathrm{M}^{\mathrm{gr}}$ Philippe Belliveau, EV., 18 juin 1913, p. 5.

${ }^{16} \mathrm{M}^{\mathrm{gr}}$ Patrice-Alexandre Chiasson, Sermon prononcé à la messe d'ouverture du $2^{e}$ Congrès de l'A.A.E. à Memramcook, 5 août 1940. CEA, Fonds A.A.E., Dossier 29-10-6.

${ }^{17} \mathrm{M}^{\mathrm{gr}}$ Patrice-Alexandre Chiasson, Ibid.

${ }^{18}$ L.C. , no 28,22 août 1940 . 
À tous ses prêtres il demande d'encourager moralement et financièrement, si nécessaire, tous les instituteurs et institutrices afin qu'ils s'inscrivent aux cours de perfectionnement pédagogiques organisés par l'Université St-Joseph. Selon lui ces cours sont peut-être le seul moyen de travailler au relèvement de la petite école ${ }^{19}$. Mais c'est l'éducation des adultes qui a été la grande priorité de $\mathbf{M}^{\mathrm{g}^{\mathrm{r}}}$ Melanson. Les Acadiens ne lisent pas et c'est là un des obstacles majeurs à leur avancement dans tous les domaines.

Nous ne lisons pas suffisamment au foyer et c'est, à mon sens, la triste mais réelle raison de notre peu de progrès jusqu'à date vers une vie religieuse plus raisonnée, une vie sociale mieux comprise et une vie économique plus pratique. ${ }^{20}$

L'Archevêque de Moncton va chercher à promouvoir partout les cercles d'études, les cours du soir, la lecture des journaux. Il voit en particulier dans les cercles d'études le moyen par excellence pour former des coopérateurs, pour aider les cultivateurs à sortir de leurs vieilles routines, pour permettre aux parents de prendre conscience de leur rôle dans l'oeuvre de l'éducation et à tous les citoyens de se familiariser avec les grandes questions sociales et religieuses de l'heure ${ }^{21}$. C'est encore pour promouvoir l'éducation des adultes que $\mathrm{M}^{\mathrm{gr}}$ Melanson fondera le journal L'Ordre social, qui aura comme premier objectif le relèvement social et catholique, et qu'il appuiera la création de l'École des sciences sociales et économiques de Moncton. Il impose à tous ses jeunes prêtres de s'inscrire à ces cours. Il lance une invitation pressante aux professionnels et aux leaders laïques d'en faire autant ${ }^{22}$. Si les Acadiens ont réussi à prendre leur place dans à peu près tous les domaines de la vie publique au Nouveau-Brunswick, ils le doivent pour beaucoup au fait que les chefs de file du siècle dernier ont fait de l'éducation leur grande priorité.

\section{Noblesse et fierté}

Privés pendant près d'un siècle des droits élémentaires de la personne humaine, peut-on se surprendre que les Acadiens aient si profondément intériorisé la peur de l'Anglais, la timidité, un manque de confiance invétéré en eux-mêmes comme personne et comme peuple et

\footnotetext{
${ }^{19}$ L.C., no 25, 2 février 1940; L.C. no 23, 31 décembre 1939.

${ }^{20}$ L.C., no 18,1 janvier 1938 .

${ }^{21}$ L.C., no 9, 31 décembre 1937; L.C., no 23, 31 décembre 1939.

${ }^{22} L . C$., no 22,19 octobre $1939 ;$ L.C., no 23,31 décembre $1939 ; L . C$., no 25,2 février 1940.
} 
qu'ils aient été arriérés dans tous les domaines. Selon $\mathrm{M}^{\mathrm{gr}}$ François Bourgeois, l'étonnant ce n'est pas que les Acadiens aient été arriérés; «ce qui est étonnant, c'est qu'ils le soient si peu!" ${ }^{23}$

Ces graves handicaps psychologiques ont vite été perçus comme les pires ennemis du relèvement national. Les leaders acadiens leur mèneront une lutte sans merci. Le Père Mombourquette, curé de Magarie au Cap-Breton, compare les hésitations du peuple acadien aux peurs de l'enfant qui voudrait faire ses premiers pas.

Le développement d'un peuple est soumis aux mêmes lois que celui d'un individu. Un peuple qui fait les premiers pas dans la voie du progrès ressemble à l'enfant qui commence à marcher. Comme l'enfant il hésite, il chancelle; ses pas ne sont pas assurés; il se défie de lui-même. Ce défaut ne fait pas grand tort à un enfant, mais à un peuple il fait un mal incalculable. Mes frères, il faut l'avouer, le peuple acadien souffre de ce mal. Si je comprends bien la pensée intime d'un grand nombre de mes compatriotes, j'y lis le mot défiance. Les autres peuples s'emparent avec avidité de tout ce que la science et la civilisation met à notre portée: le peuple acadien languit piteusement en arrière. Nous manquons de confiance. ${ }^{24}$

Avec acharnement, les leaders chercheront à convaincre les Acadiens de leur noblesse et de leur dignité en leur rappelant les pages glorieuses de leur histoire et l'étonnante résurrection de leur peuple, grâce au courage et à l'audace des ancêtres et aux attentes de Dieu sur l'Acadie.

Cessons donc de courber le front! De la fierté qui nous fasse relever le regard pour regarder les autres les yeux dans les yeux! Nous ne sommes pas des tolérés. Nous descendons d'une race d'apôtres et de chevaliers, nous avons ouvert ce pays à la civilisation, nos coutumes sont sanctionnées par les traités. Nous apportons un cachet spécial à la civilisation canadienne. Nous n'avons pas d'apologie à adresser, nous sommes fiers de notre race! ${ }^{25}$

Les Acadiens doivent donc se convaincre qu'ils ont leur place au Canada et que sans eux le pays et le monde seraient moins beaux. Ils

\footnotetext{
${ }^{23} E V$., 23 janvier 1940, p. 3.

${ }^{24}$ A.E. Monbourquette, Sermon prononcé le 15 août 1902, MA, 11 septembre 1902, p. 1.

${ }^{25} E V$., 7 avril 1915, p. 1.
} 
sont différents même des Québécois avec qui ils ont tant de liens. Conscients de cette dignité, ils se débarrasseront de cette peur qui les paralyse et ne se contenteront plus «de miettes» à la table du Canada ${ }^{26}$. Pour $\mathbf{M}^{\mathrm{gr}}$ Philippe Belliveau, curé de Grande-Digue de 1896 à $1933^{27}$, les Acadiens devront apprendre à revendiquer tous leurs droits et ils afficheront leur identité en parlant fièrement leur langue en toute occasion.

En d'autres termes notre plus grand désir est qu'à partir de ce juur vous soyez fiêrs d'êtré Acadiens. L'Acadicn vaut un autre homme, quelque soit sa nationalité ses mérites et ses titres, et il n'a pas besoin de courber son front devant qui que ce soit. L'acadien de coeur au lieu de rougir de sa langue maternelle, la cultivera et la parlera dans toutes les occasions... Il la parlera invariablement dans la famille et sur la rue, la fera apprendre à ses enfants à l'école, et ne parlera l'anglais qu'aux Anglais pour se faire comprendre dans les affaires, et ce sera toujours comme concession à l'ignorance de son auditeur. ${ }^{28}$

Pour sa part, $\mathbf{M}^{\mathrm{gr}}$ Stanislas Doucet affirme en 1912 que la majorité des Acadiens tiennent à leur langue maternelle, la parlent habituellement entre eux et, que bien qu'un très grand nombre connaisse la langue anglaise c'est l'infime minorité qui parle habituellement cette langue ${ }^{29}$. Mais il regrette cependant que les Acadiens acceptent trop facilement de parler l'anglais avec leur concitoyens de langue anglaise. Il voit là une raison pour laquelle si peu d'anglophones apprennent le français. Sur ce point comme sur bien d'autres, les Acadiens se montrent beaucoup trop complaisants à l'égard des Anglais. «Ils font trop souvent des saluts qui ne sont pas rendus» ${ }^{30}$.

$\mathrm{M}^{\mathrm{gr}}$ François Bourgeois se fait plus violent. Pour lui, les Acadiens doivent éviter de prendre pour de la vertu ce qui est souvent lâcheté et «à-plat-ventrisme" face à un certain pouvoir et au «bon-ententisme» à tout prix.

\footnotetext{
${ }^{26} E V ., 15$ avril 1932, p. 4.

$\mathrm{M}^{\mathrm{gr}}$ Stanislas-J. Doucet, "Discours à la première Convention", in Conventions nationales, t. I, p. 45-50.

${ }^{27}$ Fernand Arsenault et Edmour Babineau, " $\mathrm{M}^{\mathrm{gr}}$ Philippe Belliveau (1861-1933) et l'avenir des Acadiens", in La Société canadienne d'histoire de l'Église catholique, t. 48 (1981), p. 17-35.

${ }^{28}$ CEA, Fonds, 24, 31-1, p. 40.

${ }^{29} C E A, F .1451 A$, p. 590.
} 
Il n'y a que les peuples esclaves qui rougissent de leur nationalité ou qui se risquent à la confesser seulement avec force apologies, afin de ne point faire éclater la colère du fanatisme.

Et ce qui pousse au paroxysme de l'indignation, c'est que ces esclaves essayent de masquer leur servilité et leur lâcheté sous la belle et nécessaire vertu de prudence.

Comme si c'était de la prudence d'atrophier les sentiments de dignité, de respect de soi, pour quelques petits avantages temporels et immédiats ou pour recevoir quelques compliments sur la longueur de vue.

Oh! les vulgaires traficants du patrimoine national d'une race ! ${ }^{31}$

Ce manque de fierté nationale a donc été longtemps un grand obstacle au progrès des Acadiens. En 1905 les membres de la Société nationale l'Assomption de la région de Moncton plaçaient l'éducation à la fierté nationale comme leur premier objectif pour stopper l'anglicisation des Acadiens ${ }^{32}$. Pour sa part $\mathrm{M}^{\mathrm{gr}}$ Stanislas-J. Doucet, lors de la bénédiction du Monument à Notre-Dame-de-l'Assomption à Rogersville, en 1912, n'hésitait pas à faire intervenir nul autre que la patronne des Acadiens pour inviter ses concitoyens à réintégrer le chemin de la dignité et du progrès.

Est-ce que ce monument ne nous dit pas, ne nous crie pas "En avant?...» Est-ce que vous n'entendez pas la douce voix de Celle qui vous parle derrière le voile symbolique et qui vous dit, qui nous dit à tous: Acadiens, montez, montez toujours; ne rampez jamais. Élevez le front et marchez comme doivent marcher les fils de héros, de confesseurs de la foi! En avant! gravissez la montagne du véritable progrès, du progrès qui est bien, qui est beau, qui élève, qui ennoblit, qui ajoute une valeur réelle... à la vie. C'est par là que vous vous montrerez dignes de ma maternelle et puissante protection. ${ }^{33}$

Les Acadiens d'aujourd'hui ne pèchent pas encore par suffisance, excès de fierté et de confiance en eux-mêmes. Mais ils sont de plus en plus nombreux les citoyens, les institutions et les régions acadiennes qui arborent fièrement le drapeau de leur identité. Nous croyons que

\footnotetext{
${ }^{30}$ EV., 3 juillet 1912 , p. 4.

${ }^{31}$ EV., 26 août 1915, p. 1.

32 Henri-P. Leblanc, CEA, Fonds H.P.L. , 24.3-9.

${ }^{33} C E A$, F. 1451A, p. 585.
} 
l'Église en Acadie a droit à sa part de reconnaissance devant cet heureux cheminement vers la liberté, vers l'épanouissement personnel et national.

\section{Maîtres chez-nous}

La fierté personnelle et nationale s'enracine dans l'histoire et dans une vocation humanitaire mais elle a besoin d'un projet bien défini et de réalisations concrètes. Les Acadiens l'ont compris assez tôt. Les conventions nationales ont tracé les grandes lignes du projet acadien et $\mathrm{M}^{\mathrm{gr}}$ Philippe Belliveau le décrivait dans les termes suivants lors de la fête nationale de 1901 :

Notre idéal et notre ambition, à nous Acadiens français c'est de nous rendre dignes de nos glorieux ancêtres, de réparer les désastres de la déportation... de reprendre, pacifiquement, une partie du patrimoine dérobé par des ennemis séculaires, travailler jusqu'au bout à ce travail, lent mais sûr, d'expansion dans ce pays même qui a été le thêâtre de nos malheurs - et de reprendre notre place au soleil dans toutes les sphères accessibles aux humains. La lutte est pénible, messieurs, et les obstacles difficiles à surmonter et nous avons besoin de tout ce qui est nôtre pour ne pas faillir à la tâche. ${ }^{34}$

Pour reprendre leur place au soleil, pour devenir maîtres chez-eux, les Acadiens ont d'abord été invités à reconquérir le sol par l'agriculture et la colonisation. Selon les leaders du temps, les maîtres du pays ce sont les agriculteurs. Les Acadiens doivent donc cesser leur triste manie de se mettre au service des étrangers et d'exploiter au profit de ceux-ci les richesses industrielles du pays. Ils doivent eux-mêmes devenir les maîtres du sol ${ }^{35}$.

En plus de posséder le sol, les Acadiens devront devenir les ouvriers de leur propre demeure. Selon $\mathrm{M}^{\mathrm{gr}}$ Philippe Belliveau, "si nous sommes pour nous-mêmes incapables de faire nos affaires et de défendre nos droits, ces affaires seront mal administrées et ces droits seront méconnus» ${ }^{36}$.

Les Acadiens devront donc s'habituer à compter d'abord et avant tout sur eux-mêmes. Ils possèdent en eux la vitalité et la force

${ }^{34} E V ., 19$ septembre 1901 , p. 2.

${ }^{35} \mathrm{M}^{\mathrm{gr}}$ Philippe Belliveau, CEA, Fonds, 24, 3-2, p. 53.

${ }^{36}$ CEA, Fonds, 24, 3-2, p. 13. 
nécessaires pour travailler à leur propre relèvement. Ils l'ont déjà démontré. Les progrès réalisés se continueront s'ils demeurent étroitement unis et gardent bien vivante la flamme du patriotisme. Dès 1881 , c'était là le mot d'ordre proposé par Stanislas Doucet, alors curé de Pokemouche au Nouveau-Brunswick.

Que notre croissance soit naturelle, non artificielle, et que notre avancement en tous sens soit en raison de notre énergie et de nos propres efforts. Il ne faudra pas par conséquent fixer notre point d'appui ailleurs que sur notre propre terrain, ni baser en dehors de nous-mêmes notre espoir en l'avenir... tout en témoignant notre gratitude pour les bienfaits reçus, et en nous montrant désireux d'un coup d'épaule de temps à autre... Si nous comptions trop sur les autres, nous n'aurions jamais qu'un esprit de faiblesse et de dépendance, lequel entraverait jusqu'à un certain point notre marche et nous empêcherait d'apprendre à compter sur nousmêmes. Nous avons déjà été obligés de faire fond sur nos propres ressources, quelques faibles qu'elles puissent être, et nous le serons encore en maintes circonstances. Ce sont la force et le courage que nous déployerons et les succès que nous remporterons nous mêmes qui feront notre gloire et notre réputation. Il vaudrait mieux pour quelque temps encore ne brûler que de la chandelle plutôt que d'aller nous éclairer à la lampe à plusieurs becs de fortunés voisins, fussent-ils nos plus proches parents! ${ }^{37}$

Les Acadiens acceptèrent le défi. Ils se mirent à revendiquer avec force et ténacité leurs droits et leur part de considération et de pouvoir dans la société civile et dans l'Église. Ils se donnèrent des députés dans les assemblés législatives et au Parlement d'Ottawa et exigèrent la nomination de sénateurs et de juges acadiens. On connaît bien les luttes longues et audacieuses qu'ils menèrent jusqu'à ce que Rome leur accorde des évêques acadiens dans chacune des grandes régions de l'Acadie. Et selon $\mathbf{M}^{\mathrm{gr}}$ Philippe Belliveau, la fierté nationale a été «la principale raison» qui a poussé les Acadiens à demander un et des évêques choisis parmi les leurs ${ }^{38}$. C'est cette même fierté et ce souci de se prendre en main qui a suscité la création de communautés religieuses acadiennes, de plusieurs journaux francophones et de nombreuses institutions dans le domaine scolaire et économique. Dans toutes ces réalisations, clergé et laïcat ont travaillé en étroite collaboration ${ }^{39}$. Les progrès réalisés par

\footnotetext{
${ }^{37} M A, 8$ septembre 1881, p. 2.

${ }^{38}$ CEA, Fonds 24, 3-1, p. 15-16.

${ }^{39}$ Alexandre-J. Savoie, Un demi-siècle d'Histoire acadienne Dr. A.-M. Sormany (1885-1970), Montréal, 1976 et Un siècle de revendications scolaires au NouveauBrunswick 1871-1971, vol. I et II, Montréal, 1978.
} 
les Acadiens ces dernières années montrent bien qu'ils sont de plus en plus déterminés à prendre la parole à la table des nations, à revendiquer tous les droits et à devenir les principaux artisans de leur avenir.

\section{Quittons les vieux sentiers}

Dès le milieu du XIX ${ }^{e}$ siècle, les Acadiens sont placés devant de nouveaux défis. La société nord-américaine s'ouvre de plus en plus au monde de l'industrie et de l'urbanisation. Les Acadiens ne tardèrent pas à prendre conscience que cette société industrielle allait révolutionner profondément leurs traditions et leur vie et qu'il fallait se préparer à y entrer de plain-pied. Déjà des centaines de jeunes quittaient ces petits villages qui ne les faisaient plus vivre pour aller s'embaucher dans les manufactures américaines. On était en train de saigner l'Acadie de ses forces les plus vives, risquant ainsi de compromettre à tout jamais le projet acadien. $\mathrm{M}^{\mathrm{gr}}$ Philippe Belliveau en était convaincu:

Ah! mes chers compatriotes, si vous sondiez la grande douleur de la Patrie lorsqu'elle voit s'éloigner ses enfants! Ce sont les défenseurs qui s'en vont, ses défricheurs, ses ouvriers, les artisans de sa grandeur et de sa prospérité qui vont donner à l'étranger la force de leurs bras, la somme de leur intelligence, et, le dirais-je, jusqu'au sang de leur coeur, car beaucoup y laissent leur santé et leur vie... ${ }^{40}$

Selon $\mathbf{M}^{\mathrm{gr}}$ Philippe Belliveau, le meilleur moyen de faire cesser ces hémorragies funestes, c'était de créer en Acadie des conditions de vie à peu près égales à celles du pays voisin. Pour y arriver, l'éducation, nous l'avons déjà souligné, demeurait le moyen par excellence de se préparer aux exigences de cette société où les sciences, les finances et l'exploitation manufacturière prennent de plus en plus de place. Les participants à la Première Convention Nationale, en 1881 , se fixèrent comme objectif d'éveiller la population à l'importance des sciences appliquées dans les écoles et les collèges acadiens, afin que dans l'avenir le monde industriel «ne soit pas laissé exclusivement aux mains de l'étranger" ${ }^{41} . \mathrm{M}^{\mathrm{gr}}$ Stanislas Doucet, qui a certainement marqué de son esprit scientifique les délibérations de la Première Convention, enseignait partout que l'avenir des Acadiens allait dépendre du support du plus grand nombre possible à la cause de leur progrès intellectuel et industriel ${ }^{42}$.

\footnotetext{
${ }^{40} M A, 31$ août 1905 , p. 1.

${ }^{41}$ Conventions nationales, t. I, p. 121.

${ }^{42} M A, 23$ juin 1881 , p. 2.
} 
Pour prendre leur place dans la société industrielle, les Acadiens devaient donc moderniser leur système d'éducation, encourager le développement de l'agriculture, des pêches et de l'industrie. Plusieurs prêtres passèrent de la parole aux actes. Dès 1864, l'abbé Antoine Belcourt fondait à Rustico, sur l'île du Prince-Édouard, une Banque des fermiers dans le but de soustraire ses paroissiens aux exploiteurs et aux usuriers. Le Père Fiset, curé de Chéticamp de 1875 à 1909, qui avait juré de détruire l'empire tyrannique des Robin dans sa paroisse, se fit lui-même marchand, acheteur de poisson, président d'une mine de plâtre et propriétaire d'un grand bateau à vapeur. Les Pères Marcel-François Richard de Rogersville, François-Xavier Michaud de Bouctouche, Camille Lefebvre de Memramcook et Stanislas Doucet de Grand-Anse contribuèrent à la création de moulins à bois et de fromageries, suscitèrent des méthodes plus modernes en agriculture et travaillèrent à l'obtention de nouvelles voies ferrées pour desservir agriculteurs, pêcheurs, bûcherons et petits industriels ${ }^{43}$.

Pour sa part, $\mathbf{M}^{\mathrm{gr}}$ Philippe Belliveau ne se gênait pas pour contester ouvertement les gouvernements; selon lui, ils ne faisaient rien pour aider le développement industriel au Nouveau-Brunswick. Le curé de Grand-Digue invitait les autorités gouvernementales à cesser d'investir de fortes sommes d'argent pour amener au pays des immigrants; il leur recommandait de chercher plutôt à promouvoir les petites industries et manufactures dans les différentes régions des Maritimes ${ }^{44}$. Les coopératives et les caisses populaires ont vite été perçues comme d'excellents moyens pour faciliter aux Acadiens leur entrée dans cette nouvelle société. $\mathbf{M}^{\mathrm{gr}}$ François Bourgeois aurait vu d'un bon oeil l'organisation de syndicats professionnels pour les agriculteurs, les mineurs, les pêcheurs, les ouvriers de divers métiers et les commerçants. Mais comme à l'époque les esprits n'étaient pas encore prêts à accepter les unions syndicales, et «en attendant que cela puisse s'accomplir, il faut lutter avec les armes que nous possédons: les coopératives" ${ }^{45}$.

Les trois premiers évêques acadiens ont appuyé de toute leur autorité et de leur engagement l'essor de la Société mutuelle l'Assomption, des coopératives et des caisses populaires en Acadie. $\mathrm{M}^{\mathrm{gr}}$ Melanson enseignait pour sa part que les agriculteurs avaient tout avantage à se syndiquer. Il souhaitait vraiment les voir tous s'organiser en coopéra-

\footnotetext{
${ }^{43}$ Anselme Chiasson, "Le clergé et le réveil acadien 1864-1960", in La Revue de l'Université de Moncton (1978), p. 38-9.

${ }^{44} M A, 31$ août 1905.

${ }^{45}$ L'Ordre social, 21 nov. 1939, p. 3.
} 
tives agricoles. Des prêtres sont nommés pour promouvoir le mouvement coopératif et des quêtes spéciales sont décrétées pour lui venir en aide. Chaque nouvelle coopérative est saluée comme une victoire. En $1939, M^{\mathrm{gr}}$ Melanson annonce avec fierté que le seul diocèse de Moncton compte maintenant douze coopératives de pêcheurs et que celles-ci prédisent «une vie de réconfortante délivrance» susceptible de réparer les erreurs du passé ${ }^{46}$.

La nouvelle société industrielle et capitaliste promettait une vie meilleure aux Acadiens des Maritimes mais elle apportait en même temps de profondes transformations dans leurs coutumes et rendait souvent leurs conditions de travail quasi insupportables. $\mathbf{M}^{\mathrm{gr}}$ Patrice-Alexandre Chiasson s'est fait le défenseur de la dignité humaine dans le travail. Il s'est souvent porté à la défense des droits et devoirs de l'ouvrier comme du patron. À plusieurs reprises il a sévèrement mis en garde les patrons qui profitent d'une situation de chômage critique pour souspayer leurs ouvriers. Il a encouragé les associations d'ouvriers (surtout les associations catholiques), les organisations de patrons et la mise sur pied de commissions mixtes dans lesquelles ouvriers et patrons discuteraient de leurs intérêts particuliers et réciproques pour en arriver à des ententes utiles au bien de tous ${ }^{47}$.

Pour $\mathrm{M}^{\mathrm{gr}}$ François Bourgeois, c'est surtout la théorie d'Adam Smith sur la division du travail qui, malgré bien des avantages, devient une menace de plus en plus lourde à la dignité humaine. Elle dégrade l'intelligence de l'ouvrier, détruit sa satisfaction personnelle et accentue sa dépendance face au patron et à l'industrie. C'est là un problème sérieux sur lequel on devrait se pencher dans des rencontres patrons-ouvriers. Devant tous ces défis, $\mathbf{M}^{\mathrm{gr}}$ Bourgeois, en toute fidélité aux priorités acadiennes, invite ses compatriotes à s'inscrire dans les écoles techniques et à s'imposer partout par leur compétence ${ }^{48}$.

Cette compétence, $\mathrm{M}^{\mathrm{gr}}$ Arthur Melanson la réclame également pour les agriculteurs. "Quand donc ces petits agriculteurs de misère qui composent les presque totalité de notre population quitteront enfin les vieux chemins battus de la routine pour se donner une fois pour toutes à des méthodes plus éclairées, plus pratiques et plus efficaces?» ${ }^{49}$ Selon le

\footnotetext{
${ }^{46}$ L.C. , 31 décembre 1937, 1 janvier 1939;

$M^{\mathrm{gr}}$ Patrice-Alexandre Chiasson, 22 avril 1936.

${ }^{47}$ L.C. , 24 février 1927 et 18 janvier 1934.

${ }^{48} E V$, 23 janvier 1940, p. 3.

${ }^{49}$ L.C. , 1 janvier 1939.
} 
premier archevêque de Moncton, la vraie crise des années 30 était surtout d'ordre moral, social et religieux. Le cancer à détruire était le pessimisme, l'individualisme et l'indifférence de trop nombreux chrétiens et Acadiens. C'est là le grand mal qui ronge les hommes, ruine la famille et la société. Il fallait tout mettre en oeuvre pour changer cette immense armée de blasés et d'indifférents pour qui «il n'y a plus rien à faire». En prêchant leur paresse ils deviennent de véritables éteignoirs; ils sèment la mort et ils la récoltent abondamment ${ }^{50}$. Pour l'Archevêque de Moncton, il faut prêcher aux Acadiens confiance et optimisme, réveiller "cette armée de grands garçons à ne rien faire", les inciter à sortir de leur torpeur et les orienter vers de nouvelles avenues, grâce aux différents programmes d'études offerts dans la Province. C'est par la compétence que les Acadiens prendront la place qui leur revient dans une société en pleine transformation ${ }^{51}$.

Avec les moyens très pauvres dont disposaient les Acadiens jusqu'au milieu du $\mathrm{XX}^{\mathrm{e}}$ siècle, nous croyons que ceux-ci ont réussi malgré tout à se tailler une place respectable dans le monde de la finance et de la petite industrie. Nous pouvons affirmer sans hésitation que le clergé et l'épiscopat acadien, en collaboration bien sûr avec des laïques de plus en plus nombreux, ont grandement contribué à ce relèvement économique.

\section{CONCLUSION}

Quel a été l'impact de l'Église en Acadie de 1864 à 1942? La recherche actuelle permet de répondre à cette question de façon satisfaisante bien que plusieurs personnes attendent toujours l'occasion de prendre la parole à la barre des témoins. Le rôle des laïques, de beaucoup d'autres acteurs de la vie acadienne, de la femme en particulier et des communautés religieuses devra être davantage mis en lumière. Ce travail progresse lentement. Mais déjà il est possible de conclure avec suffisamment de preuves à l'appui que l'Église catholique a eu un impact salutaire sur la reconstruction de l'Acadie. Malgré un certain nombre de contre-témoignages, elle a contribué de façon décisive à la reconquête de l'identité personnelle et nationale des Acadiens ${ }^{52}$.

\footnotetext{
${ }^{50}$ L.C., 22 février 1937.

${ }^{51}$ L.C., 22 février 1937 et $L . C ., 31$ décembre 1937.

52 Avec mon collègue Edmour Babineau, je publierai bientôt une série d'études qui montreront de façon plus précise les aspects négatifs et positifs de l'impact de l'Église en Acadie.
} 
Pour servir le projet acadien, le clergé, l'épiscopat et les laïcs ont oeuvré sur de nombreux plans, ce qui a multiplié bien sûr les possibilités d'erreurs et d'échecs. Mais leur idéal d'égalité, de liberté et de fraternité, le peuple l'a poursuivi sans relâche et avec des gains remarquables de 1864 à 1942, pour ne mentionner que la période étudiée. Les quatre mots d'ordre: L'éducation notre salut, Noblesse et fierté, Maîtres chez-nous et Quittons les vieux sentiers ont catalysé de nombreuses énergies, beaucoup de créativité et d'héroïsme et sont devenus les points d'appui de l'Acadie contemporaine. İis ont servi de fer de lance à la Société Nationale l'Assomption, à l'Action catholique, aux diverses commanderies de l'Ordre de Jacques Cartier, à l'Association Acadienne d'Éducation au Mouvement Coopératif, à plusieurs institutions religieuses, sociales, politiques et culturelles. Les arts, la littérature, les sciences et l'économie ne seraient pas chez-nous ce qu'ils sont si des générations de prophètes n'avaient pas périodiquement réveillé de leurs appels à la vie les ossements désséchés d'un peuple qui avait toutes les raisons de mourir.

En Acadie, des hommes et des femmes, animés d'une profonde confiance dans ce qu'ils avaient perçu comme le projet du Christ, ont cru fermement que le Canada et l'humanité seraient moins beaux si les Acadiens ne faisaient pas entendre cette Parole unique qu'ils ont reçue en héritage et qu'ils ont mission de manifester à leurs frères et soeurs du monde. Ne pourrait-on pas souhaiter, en terminant, que les Acadiens de toute génération puissent aujourd'hui se mobiliser à nouveau derrière les quatre mots d'ordre que leur ont légués leurs devanciers, afin de faire naître, et de manifester partout avec force et tendresse cette Parole unique qu'ils portent en eux, mais qu'ils ne possèderont véritablement que dans le don.

Fernand Arsenault

Département de sciences

religieuses

Université de Moncton 